\title{
Coordinating care in acute diabetes foot problems: no mean feet!
}

\author{
SHAHLAA WALSH, ROSELLE HERRING, HELEN GRIFFITH, JULIE KOHLS-GATZOULIS, SUE DAVIDSON
}

\begin{abstract}
A multidisciplinary foot care team exists to coordinate specialist care for people with complex diabetes-related foot problems. The local multidisciplinary diabetes foot care team (MDFT) consists of a diabetes specialist, diabetes specialist nurse, orthopaedic surgeon, a visiting vascular surgeon and a podiatrist. Concerns around inpatient care were raised following a man who presented with a foot attack who underwent major limb amputation and subsequently died. A root cause analysis and Serious Untoward Incident panel identified reasons for delays in good clinical care. Undue emphasis on the need for further imaging, lack of urgency to surgically debride and request for other specialty assessments contributed to the delay in providing potentially life-saving treatment. Specialist ownership of patients with multidisciplinary input and lack of a clear management pathway also contributed. The Guildford Footpath is a clinical decision tool for acute diabetes foot problems developed by physicians and surgeons working together. It is a practical and safe way of helping non-specialists to assess patients presenting with acute diabetes foot problems. It clearly assigns responsibilities amongst specialist teams and provides a route for each specialist team to access members of the MDFT in a timely manner. It has streamlined care and given those involved more confidence in making decisions. We hope that ongoing audit will show a reduction in length of stay and amputation rates.
\end{abstract}

Br J Diabetes 2016;16:65-68

Key words: diabetes mellitus, diabetic foot disease, serious untoward incident, amputations, Guildford Footpath

\section{Introduction}

Foot problems in people with diabetes have a significant financial impact on the NHS through primary care, community care, outpatient costs, increased bed occupancy and prolonged stays in hospital. A report published in 2012 by NHS Diabetes estimated that around $£ 650$ million (or $f 1$ in every $£ 150$ the NHS

Royal Surrey County Hospital, Guildford, Surrey, UK

Address for correspondence: Dr Roselle Herring

Centre for Endocrinology, Diabetes and Research, Royal Surrey County

Hospital, Egerton Road, Guildford, GU2 7XX, UK

E-mail: roselle.herring@nhs.net

http://dx.doi.org/10.15277/bjd.2016.078 spends) is spent on foot ulcers or amputations each year. ${ }^{1}$

The risk of foot problems in people with diabetes is increased, largely because of either diabetic neuropathy or peripheral arterial disease or both. It is estimated that $10 \%$ of people with diabetes will have a diabetic foot ulcer at some point in their lives. Fifty percent of patients who have a diabetes-related major amputation are likely to die within 2 years, and approximately $60 \%$ of patients with diabetic ulcers are unlikely to survive more than 5 years. ${ }^{2}$

The National Diabetes Inpatient Audit in 2013 showed that, in England, $15.7 \%$ of inpatients had diabetes, with $9.1 \%$ of admissions related to diabetic foot problems. ${ }^{3}$ Our local Clinical Commissioning Group (CCG), Guildford and Waverley, reported 435 episodes of care for diabetic foot disease between 2011/12 and 2013/14, accounting for 4,889 nights in hospital. ${ }^{4}$ The length of stay can be prolonged, with associated poor clinical outcomes and increased risk of re-admission. Potential reasons are poorly coordinated investigations, interventions, consultations and care planning. ${ }^{5}$

The National Institute for Health and Care Excellence (NICE) has sought to address problems in diabetes foot management with the publication of a guideline on diabetic foot prevention and management. ${ }^{1}$ The NICE guideline recommends that one health professional should be responsible for coordinating inpatient care between specialists, the patient and other health professionals. This professional is responsible for overseeing the multidisciplinary coordination of care, scheduling relevant interventions and investigations, and ensuring appropriate and timely discharge planning. Each hospital should also have a care pathway for people with diabetic foot problems who need inpatient care.

Many trusts have implemented a multidisciplinary foot care team (MDFT) to coordinate specialist care. However, the involvement of different specialties can result in uncertainty or delay in the decision-making process. This is particularly true when not all members of the MDFT are able to see the patient simultaneously. This is even more apparent when vascular services are not on site (vascular spoke).

Implementing a foot care decision tool is a valuable aid in identifying high-risk patients as early into their admission as possible and determining responsibilities for ongoing management.

\section{Case report}

A 54-year-old male patient with type 2 diabetes and an extensive cardiac history was admitted with a suspected stroke. During the assessment the admitting medical team noticed that the patient was septic and the source was probably from an infected left foot ulcer for which he had recently finished a course of antibiotics. On examination he was pyrexial, hypotensive and had a 
Figure 1. Left foot $X$-ray showing calcified vessels and gas in the soft tissues of the heel

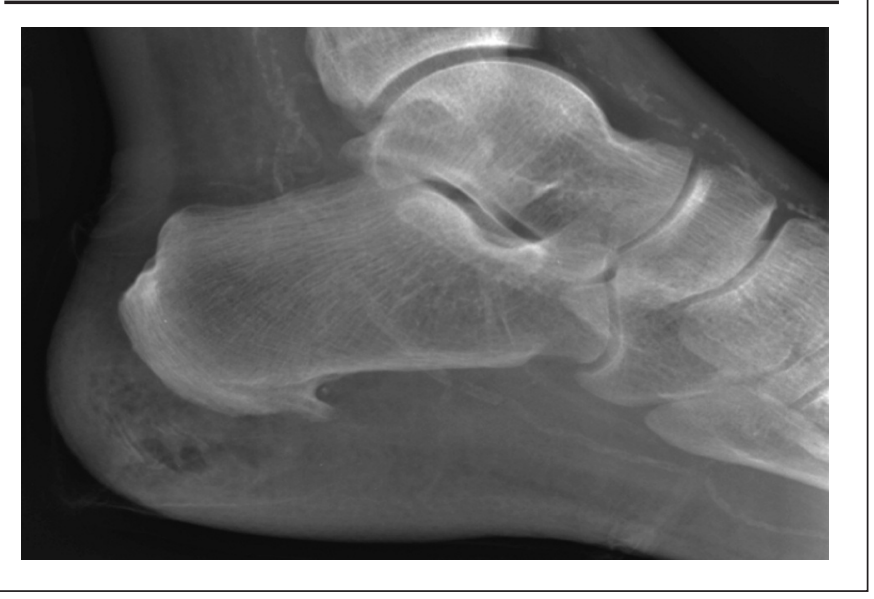

blood glucose level of $18.4 \mathrm{mmol} / \mathrm{L}$. The patient's left foot was oedematous, erythematous and hot, with a purplish hue and telangiectasia around an ulcer.

Left foot $X$-ray revealed gas in the soft tissue of the heel and calcified vessels (Figure 1). He was commenced on broad-spectrum antibiotics according to the trust's guidelines for diabetic foot infection.

The orthopaedic team reviewed the patient and advised further investigation with urgent magnetic resonance imaging (MRI) of the left foot. Several discussions between the orthopaedic team, surgical team and off-site vascular team occurred over the following $72 \mathrm{~h}$, with a view to determine who would take responsibility for the patient's ongoing surgical management. During this time the patient was transferred to intensive care for invasive monitoring and inotropic support. ITU intensivists requested a review by the diabetes team, who diagnosed wet gangrene. The patient was finally transferred the following day to the vascular centre for surgical treatment but unfortunately had a cardiac arrest in theatre. Despite this he was effectively resuscitated and transferred to the intensive care unit.

Three days later he had a left below-knee amputation and continued to require renal replacement therapy and intensive care support. Eleven weeks from the initial presentation, after further cardiac events and failure to respond to treatment, the patient died of multi-organ failure.

\section{Serious Untoward Incident}

Following this event a Serious Untoward Incident (SUI) process was initiated. The Serious Incidents Framework was created to investigate the root cause of serious untoward incidents in all NHS trusts in England. ${ }^{6}$ These SUls include 'acts or omissions in care' resulting in death, serious harm or injury or affect the trust's capacity to provide an acceptable level of care. Under the current guidelines, when an SUI occurs in an NHS organisation, a thorough root cause analysis must take place within 60 working days of the event and a report produced based on the seven principles of the Serious Incidents Framework.
As a result of root cause analysis, the Commissioner from the local CCG will ensure the final report and action plan meet requirements and are implemented. There should be opportunity to learn from the incident and information should be shared with relevant parties including patients and their families.

\section{Standard of Care for Diabetic Foot Problems}

The NICE guidelines suggest that an inpatient MDFT should thoroughly assess and manage the complications of diabetic foot disease to prevent deterioration and aid healing. Patients should be referred to the MDFT within $24 \mathrm{~h}$ of a diabetic foot problem being identified. In practice this often means one member of the MDFT seeing the patient initially. A foot care pathway is important to ensure that this rapid assessment and appropriate management is made.

\section{Lessons learnt}

The case underwent root cause analysis and the SUI panel highlighted key areas which required immediate attention to improve the standard of care.

\section{Lesson 1}

Lack of urgency was identified as one of the main shortfalls. The severity of his condition was not acknowledged by the admitting non-specialist team, so referrals made to appropriate specialties did not highlight the seriousness of his condition and resulted in a delay in appropriate assessment and management within the first $24 \mathrm{~h}$ of admission.

\section{Lesson 2}

Undue emphasis was placed on getting further imaging and other specialty assessments instead of emergency surgical intervention. Where early incision and drainage of a foot abscess is indicated, this can be undertaken by orthopaedic or vascular surgeons at registrar level. However, this opens up the possibility that both will consider it to be the other's decision and action.

\section{Lesson 3}

Taking ownership for this patient's diabetes-related foot problem was another key area which contributed to his poor management. There is a need to clarify roles and assign responsibility in managing diabetic foot emergencies. There should also be a quick resolution of differing opinions.

\section{Lesson 4}

Emergency treatment should not be delayed pending a MDFT foot round. The diabetic MDFT ward round occurs every 2 weeks at this hospital and mainly focuses on the management of outpatients with complex foot problems. Care of inpatients is via a member of the MDFT team assessing in $24 \mathrm{~h}$ and contacting the relevant colleagues to ensure coordinated care.

\section{Lesson 5}

There is a lack of a clear diabetic foot management pathway for patients presenting acutely. 
Figure 2. The Guildford Foot Pathway

THE GUILDFORD FOOTPATH - Immediate care of patients with acute DIABETES FOOT problems

Name

DOB
Hospital no

Date \& time

- Patients with diabetes must have both feet examined by a doctor on admission even if there are no symptoms (neuropathy)

- You must take down any dressings

- Subsequent daily inspection by nurses is mandatory

- The minimum assessment (RIGHT) is needed to use this pathway

\begin{tabular}{|l|}
\hline ON CALL T\&O \\
Do any of the following apply: \\
- Wet gangrene \\
- White cold pulseless foot \\
- Critically ischaemic limb
\end{tabular}

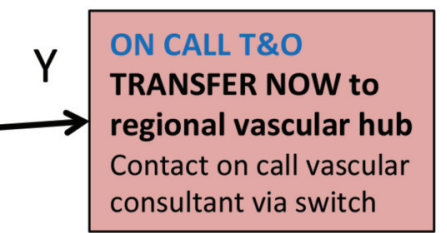

$N \downarrow$

ON CALL T\&O

Are any of the following present:

- Hot red swollen foot

- Boggy tissue when pressed

- Collection of pus on exam

- Crepitus or gas in the tissues on a plain XR

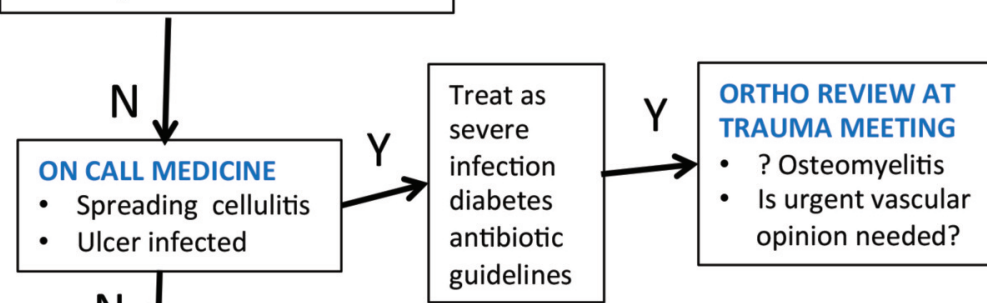

\section{$\mathrm{N} \downarrow$}

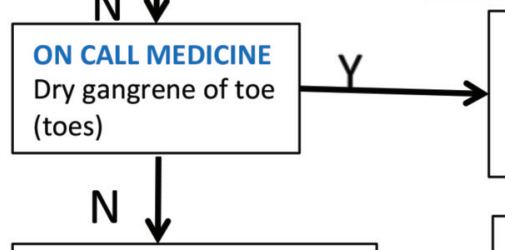

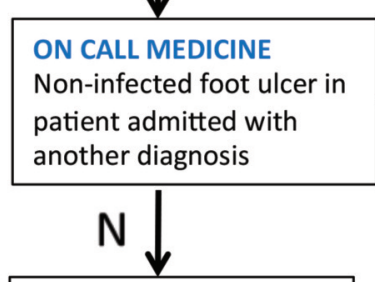

ON CALL T\&O

Warm, slightly swollen,

non infected foot

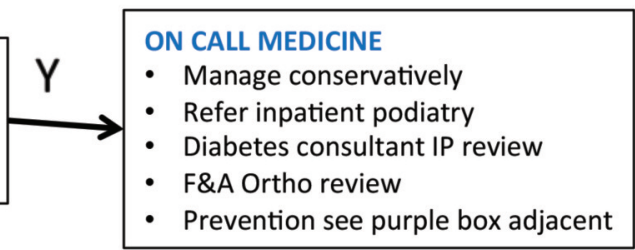

ON CALL MEDICINE

- Non-urgent (<3 days) inpatient vascular review

- DSN review if needed

\section{MEDICINE}

- Refer inpatient podiatry

- Diabetes consultant IP review

- Prevention see purple box adjacent

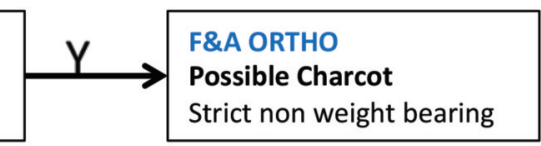

CIRCLE OUTCOME and FILE IN NOTES

Royal Surrey County Hospital W/HS

NHS Foundation Trust

Minimum assessment:
Foot pulses $R$
Sensation $R$
FBC Hb
CRP WCC
Creatinine
Plain x ray of foot

WHO LOOKS AFTER THE PATIENT?

- ON CALL T\&O = admitting ortho surgeon until T\&O meeting next working day

- F\&A ORTHO = foot and ankle ortho. Weekday rota

- ON CALL MEDICINE = admitting physician

- DIABETES = Diabetes ward

\section{ON CALL T\&O}

URGENT (within 6hrs)

incision and drainage

On call T\&O to undertake

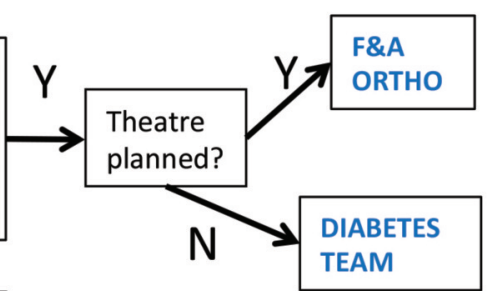

CARE OF ALL PATIENTS WITH DIABETES FOOT PROBLEMS

- Pressure mattress

- Minimal weight bearing

- No anti-embolism stockings if neuropathy or impaired pulses

- Inform diabetes registrar when patient admitted

\section{Completed by.}

Date...

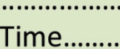

.... Time.....................

V2 generic 10.15(C) S Davidson

DSN, diabetes specialist nurse; F\&A ORTHO, Foot and Ankle Orthopaedics; T\&O, Trauma and Orthopaedics; XR, X-ray. 
Box 1. Clear guidelines produced by the trauma and orthopaedics team

The red, swollen foot in a septic, diabetic patient must be assumed to contain pus that requires emergency incision and drainage.

\section{DO NOT WAIT FOR}

- A MRI

- The next day

- The end of the CEPOD list

- The patient to be starved for 6 hours

- Vascular opinion

It is much better to incise a red, swollen, diabetic foot and find no collection than to fail to incise a diabetic foot abscess

\section{PUS UNDER PRESSURE KILLS LIMBS AND PATIENTS}

\section{Guildford Footpath}

In response to the findings, we have developed a cross-specialty clinical decision tool for acute diabetes foot problems to be used by specialist and non-specialist clinical teams. Importantly, this has been produced and ratified by both diabetologists and orthopaedic and vascular surgeons. The Guildford Footpath is shown in Figure 2, and Box 1 shows clear guidelines set by the trauma and orthopaedic team for emergency care.

Critical to this clinical decision tool is that it clarifies where the responsibility for the patient remains at each stage. Importantly, the foot and ankle orthopaedic surgeons have been involved in its development. They clarified the need for urgent surgical intervention to their non-specialist colleagues who see these patients when on call. Incision and drainage should be performed within $6 \mathrm{~h}$ and not delayed by a request for imaging or vascular opinion.

\section{How to use the Guildford Footpath}

The clinical decision tool begins with a basic essential examination technique and minimum assessment criteria to ensure that patients with diabetes-related foot problems are correctly identified. This must be performed before moving further down the clinical decision tool. It highlights in red the emergency presentations of the acute diabetic foot patient. From these red boxes, it is clear who must take responsibility for the patient and the location where they will be best managed.

The Guildford Footpath has been rolled out across local medicine, surgical and emergency specialties. It has been presented to all junior doctors, to consultants of all specialties during educational half days and at the weekly medical journal club. It is also easily available via the Trust diabetes icon, which is ideally placed on the Trust desktop and accessible to all healthcare pro-

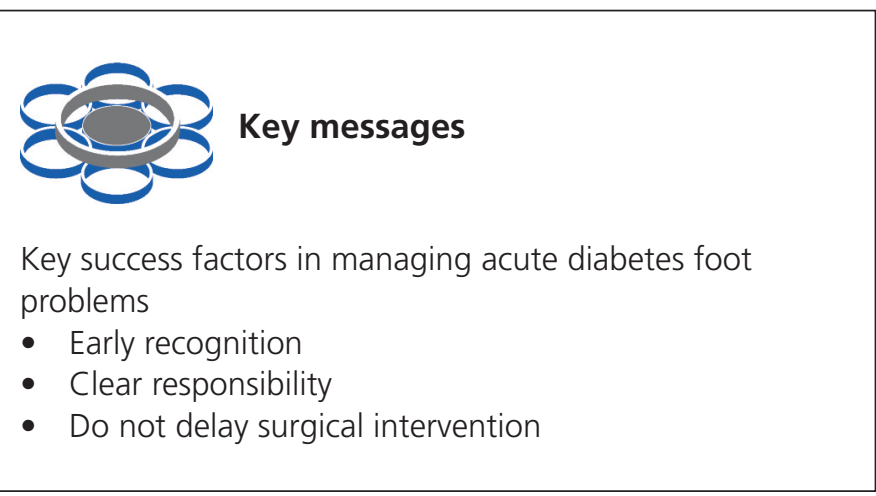

fessionals working in the Trust. We continue to audit change in practice and hope that it will translate into a reduction in length of stay, amputation and mortality rates.

\section{Conclusions}

The Guildford Footpath was developed by physicians and surgeons working together, as part of the Trust's Standardised Pathways and Clinical Excellence (SPACE) initiative. It is a practical and safe way of helping non-specialists to assess patients presenting with acute diabetes foot problems. It also clearly assigns responsibilities amongst specialist teams and provides a route for each specialist team to access members of the MDFT in a timely manner.

For our patient, the Guildford Footpath would have resulted in earlier surgical intervention. Although the outcome may not have changed, all those involved in his care would have been clear about their roles and responsibilities as well as the timing of any interventions.

This clinical decision tool has already streamlined care and given those involved more confidence in their decision-making. We hope that ongoing audit will show a reduction in length of stay, amputation and mortality rates.

\section{Conflict of interest None. Funding None.}

\section{References}

1. National Institute for Health and Clinical Excellence (NICE). Diabetic foot problems. Inpatient Prevention and Management of Diabetic Foot Problems. London: NICE, 2015.

2. All Party Parliamentary Group on Vascular Disease. Tackling peripheral arterial disease more effectively: saving limbs, saving lives. http://appgvascular.org.uk/media/reports/2014-03-tackling_peripheral_ arterial_disease_more_effectively_saving_limbs_saving_lives.pdf

3. Health and Social Care Information Centre ( $\mathrm{HSCIC})$. National Diabetes Inpatient Audit. http://www.hscic.gov.uk/diabetesinpatientaudit

4. Public Health England. Diabetes footcare activity profile. http://www yhpho.org.uk/diabetesprofilesfoot/ccg_pdfs15/09N_Diabetes_Footcare_P rofile 2015.pdf. June 2015.

5. Flanagan D, Moore E, Baker S, Wright D, Lynch P. Diabetes care in hospital — the impact of a dedicated inpatient care team. Diabet Med 2008;25(2):147-51. http://dx.doi.org/10.1111/j.1464-5491.2007.02326.x

6. NHS England. Serious Incident Framework. http://www.england.nhs.uk/ ourwork/patientsafety/serious-incident/ 